\title{
Cervical Spine Computed Tomography Imaging Artifact Affecting Clinical Decision-Making in the Traumatized Patient
}

\author{
Aaron C. Coats ${ }^{1}$, Matthew S. Nies ${ }^{*}, 2$ and David Rispler ${ }^{3}$ \\ ${ }^{1}$ American Health Network, Indy Bone and Spine, Indianapolis, IN, USA \\ ${ }^{2}$ Michigan State University, College of Human Medicine, Grand Rapids, MI, USA \\ ${ }^{3}$ River Valley Orthopedics, Grand Rapids, MI, USA
}

\begin{abstract}
CT scanning is an important tool in the evaluation of trauma patients. We review a case involving a trauma patient in which a cervical spine computed tomography (CT) artifact affected decision-making by physicians. The CT artifact mimicked bilateral dislocated facets (51-B1.1). On the basis of CT findings, the patient was transferred to a different hospital for evaluation. Discrepancy between the primary CT scan and patient physical exam prompted secondary CT scans and X-ray evaluation; neither of these studies showed osseous abnormalities. This case reinforces the necessity for physicians to formulate their diagnosis based upon multiple areas of information including physical examination, plain $x$-ray and subsequent advanced imaging, rather than relying solely on advanced imaging.
\end{abstract}

Keywords: Artifact, cervical spine, computed tomography, discrepancy, osseous abnormality, physical examination.

\section{INTRODUCTION}

History, physical examination and diagnostic imaging are integral components of the evaluation of blunt trauma. In recent years, diagnostic imaging of blunt trauma in emergency departments has become more reliant upon the use of helical computed tomography (CT) scanning. CT imaging is an efficient, sensitive and reliable method for detecting traumatic injury. Using CT, true spinal injuries are rarely missed.

However, CT-generated artifacts can interfere with clinical decision-making. This has been demonstrated by several previous reports. Sciubba et al. describe a case very similar to the case presented here in which a 15 -year-old boy who sustained a neck injury during a lacrosse game was initially diagnosed with subluxation of the cervical spine, and subsequently transferred to secondary institution where a second CT demonstrated normal bony alignment [1]. Further, Dhandapani et al. describe a case report of two patients with cervical subluxation on initial CT examination. In the first case, the artifact was noted after a subsequent lateral $\mathrm{x}$-ray was obtained and was normal. In the second case, the subsequent plain $\mathrm{x}$-ray was inconclusive but because of the experience obtained in the first case, motion artifact was still the suspected cause. A repeat CT scan was without bony abnormality [2].

We support these previous reports with a case of a trauma patient who was initially diagnosed with bilateral cervical facet dislocation. After transfer to a different hospital, physical examination of the patient prompted

*Address correspondence to this author at the Michigan State University, College of Human Medicine, Grand Rapids, MI, USA; Tel: +616 366 8885; E-mail: Matthew.Nies@gmail.com greater scrutiny of the original CT images, and secondary CT images were obtained. These scans showed no C-spine abnormalities. This case is important for a multitude of reasons. First, in busy trauma centers, it is very possible for fractures to be missed. This case emphasizes the need for integration of history, physical examination findings, as well as both radiographic and $\mathrm{CT}$ imaging in assessing a patient. Second, it is important for all treating physicians to do their own independent assessment and review of imaging, especially around shift changes and during the acute trauma period.

\section{CASE REPORT}

A 21 year-old female driver was involved in a rollover motor vehicle accident and transported to the emergency department. Physical examination was equivocal. Cerebral spine CT imaging was used to assess injury to the spine. Results indicated bilateral dislocated facets (51-B 1.1) at the level of C4-5. The patient was transferred to our tertiary care hospital for further evaluation of the spinal dislocation based on the CT findings alone.

Upon further evaluation, the patient's physical exam demonstrated no neurological deficits, with only minimal paraspinal c-spine muscle tenderness to palpation. This diagnostic discrepancy with physical examination prompted re-evaluation of the primary CT scan.

After further review of the original CT scan images, an aberration was noted on the axial image. On closer inspection, the sagittal image also appeared aberrant (Figs. 1, 2). Thus, a repeat c-spine CT (Figs. 3, 4) was ordered. These images showed no osseous abnormalities, consistent with the physical examination. A lateral c-spine $\mathrm{x}$-ray (Fig. 5) confirmed this finding. Per protocol for cervical sprain, 
the patient was placed in a Miami-J cervical collar and discharged with follow-up.

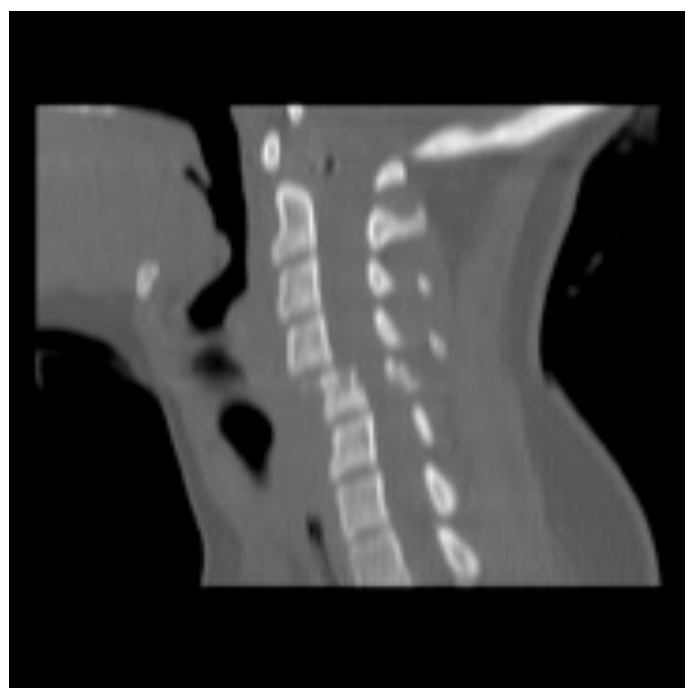

Fig. (1). Saggital cut of initial cervical spine CT scan.

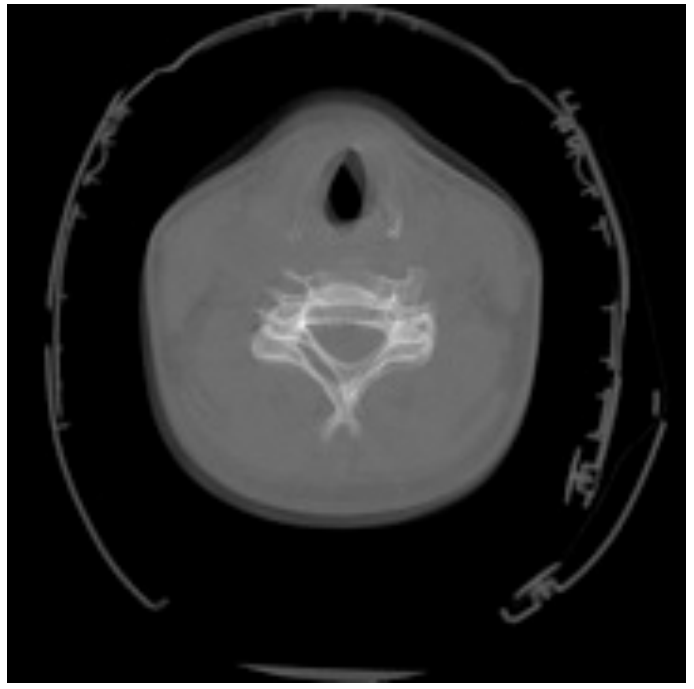

Fig. (2). Axial cut of initial cervical spine CT scan.

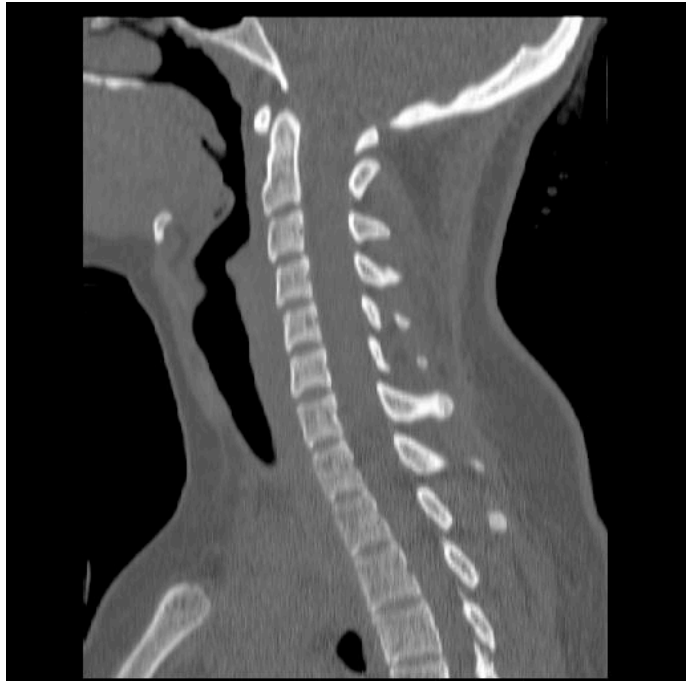

Fig. (3). Sagittal cut of repeat cervical spine CT scan.

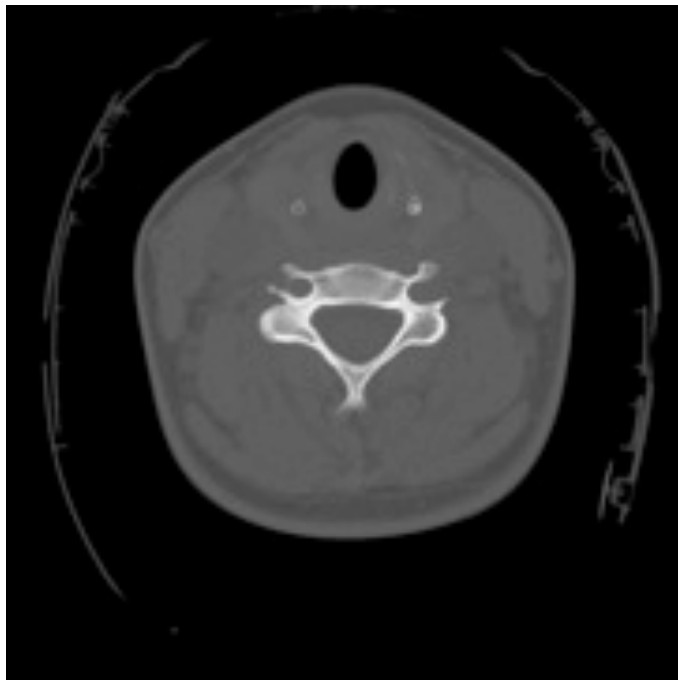

Fig. (4). Axial cut of repeat cervical spine CT scan.

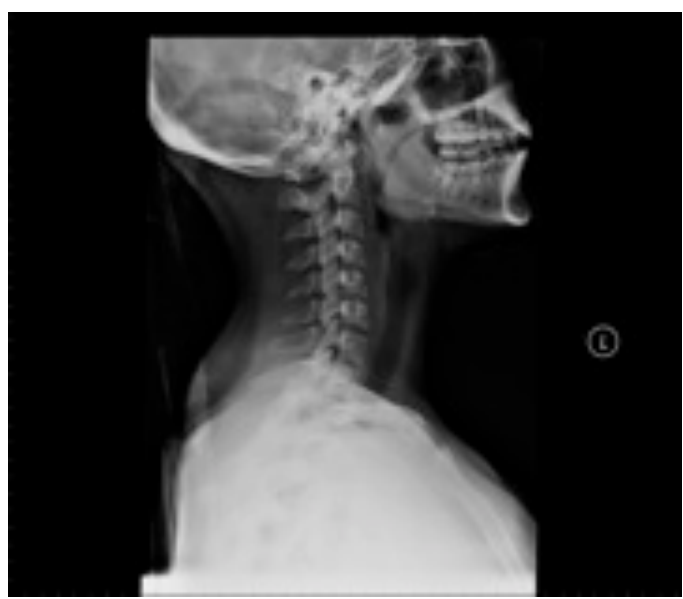

Fig. (5). Lateral x-ray confirming normal cervical spine alignment.

\section{DISCUSSION}

History, physical examination, and diagnostic imaging are integral components in the evaluation of a trauma patient. Clearing a possible c-spine injury is a high priority in trauma patients because missed diagnosis can lead to further injury. Guidelines for radiologic evaluation of the c-spine have evolved over time and are slightly different for different specialty organizations $[3,4]$. The Advanced Trauma Life Support (ATLS) guidelines recommend $\mathrm{x}$-ray examination of the c-spine, with supplemental axial CT images of areas that are suspicious or not adequately visualized with $\mathrm{x}$-ray. The three-view radiographs recommended are: lateral, anteroposterior and open-mouth odontoid c-spine radiographs (CSR) [4]. In practice, obtaining adequate visualization with CSR can be difficult. In one prospective study, radiographs were judged inadequate (by a radiologist) in about half of high-energy trauma patients [5].

In an effort to reduce cost to patients, criteria have been developed to attempt to reduce unnecessary imaging. The National Emergency X-Radiography Utilization study (NEXUS) Low Risk Criteria aid clinical decision making to rule out c-spine injury [6]. If trauma patients have no posterior midline tenderness, are alert, are not intoxicated, have no focal neurological deficit, and no painful and 
distracting injuries, then radiographs are not warranted. When these conditions are not met, adequate three-view cspine radiographs are recommended to detect possible osseous abnormalities.

Modern 64-slice helical CT has been shown to be highly sensitive and specific and is done more rapidly, in spite of the fact that it is more costly than CSR. As a result, the CT scan has become an integral modality in the evaluation of trauma patients. Several studies have shown the value of using CT scan as an adjunct to CSR to enhance the detection of fractures that are undetected by CSR in trauma patients [7, 8]. Also, CT scans are superior to CSR in severe, blunt, multiple injury trauma patients [9]. In contrast, for low risk trauma patients, CSR has been shown to be adequate to detect c-spine injury [10]. A recent prospective clinical study compared CT used alone for detecting c-spine injury in highenergy trauma patients compared to adequate three-view CSR. This study showed that CT scan was more sensitive, had a higher specificity, a higher predictive value and a lower negative predictive value compared to three-view radiographs [5]. Results from these studies and others have led to the suggestion that c-spine CT should be used as a primary evaluation tool, and thus replace CSR as a means of clearing the $\mathrm{C}$-spine in traumatized patients $[5,7,11]$.

In spite of cost and sensitivity/specificity considerations, the case discussed here demonstrates the importance of integration of the elements of the physical examination, plain $\mathrm{x}$-rays, and CT scanning, and most importantly, clinical judgment, in the final assessment of the patient. While CT scanning is a very sensitive modality for detecting spinal injury, this increased sensitivity comes at a cost of an increased false-positive rate. As such, clinicians must perform careful scrutiny of the images obtained, as well as correlation with other imaging modalities and physical examination in assessing for bony abnormality. Further, MRI may be obtained for suspicion of ligamentous injury or for a more detailed evaluation of the soft tissues, if necessary.

\section{CONCLUSION}

The case report presented here highlights a situation in which a CT scan error led to unnecessary patient transfer as well as a second CT scan, both of which being very costly. As such, this case report underscores the importance of integrating all aspects of patient history, precise physical examination, diagnostic imaging, and clinical judgment in making informed decisions in the management of alert, coherent trauma patients. Re-evaluation is necessary when the CT scan does not correlate with radiographic data or physical examination. This careful integration of physical examination and detailed review of images obtained will undoubtedly lead to a decrease in unnecessary patient transfers, imaging, and procedures in the future.

\section{ABBREVIATIONS}

CT $=$ Computed tomography

CSR $=$ Cervical spine radiography

ATLS $=$ Advanced trauma life support

\section{CONFLICT INTEREST}

The authors confirm that this article content has no conflict of interest.

\section{ACKNOWLEDGEMENTS}

Declared none.

\section{REFERENCES}

[1] Sciubba D, Dorsi M, Kretzer R, Belzberg A. Computed tomography reconstruction artifact suggesting cervical spine subluxation. J Neurosurg Spine 2008; 8(1): 84-7.

[2] Dhandapani S, Salunke P, Mukherjee K. "Artifactual fracturesubluxation" of cervical spine in computed tomography screening sans plain radiographs. Spine J 2013; 13(11): e45-8

[3] Sciubba D, Petteys R. Evaluation of blunt cervical spine injury. South Med J 2009; 102(8): 823-8.

[4] American College of Surgeons. Advanced Trauma life support for doctors, student course manual. $8^{\text {th }}$ ed. Chicago: American College of Surgeons 2008.

[5] McCulloch P, France J, Jones D, et al. Helical computed tomography alone compared with plain radiographs with adjunct computed tomography to evaluate the cervical spine after highenergy trauma. J Bone Joint Surg Am 2005; 87(11): 2388-94.

[6] Hoffman J, Schriger D, Mower W, Luo J, Zucker M. Low-risk criteria for cervical-spine radiography in blunt trauma: a prospective study. Ann Emerg Med 1992; 21(12): 1454-60.

[7] Barba C, Taggert J, Morgan A, et al. A new cervical spine clearance protocol using computed tomography. J Trauma 2001; 51(4): 652-6; discussion 656-7.

[8] Borock E, Gabram S, Jacobs L, Murphy M. A prospective analysis of a two-year experience using computed tomography as an adjunct for cervical spine clearance. J Trauma 1991; 31(7): 1001-6.

[9] Berne J, Velmahos G, El-Tawil Q, et al. Value of complete cervical helical computed tomographic scanning in identifying cervical spine injury in the unevaluable blunt trauma patient with multiple injuries: a prospective study. J Trauma 1999; 47(5): 896-902.

[10] Nguyen G, Clark R. Adequacy of plain radiography in the diagnosis of cervical spine injuries. Emerg Radiol 2005; 11(3):15861 .

[11] Blackmore C, Mann F, Wilson A. Helical CT in the primary trauma evaluation of the cervical spine: an evidence-based approach. Skeletal Radiol 2000; 29(11): 632-9. 